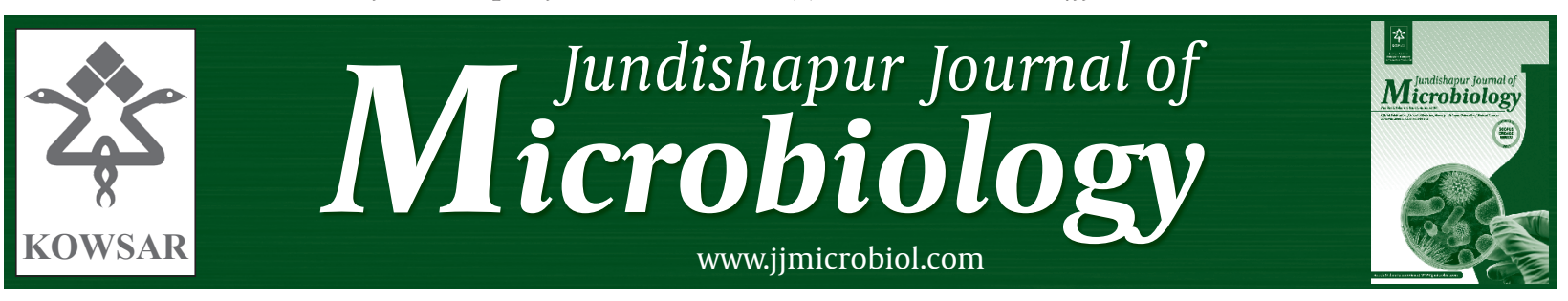

\title{
Concomitant Pulmonary Nocardiosis and Tuberculosis in a Patient With Rheumatoid Arthritis: A New View Point
}

\author{
Zohreh Aminzadeh ${ }^{1,2}$, Ilad Alavi Darazam ${ }^{3,4^{*}}$ \\ ${ }^{1}$ Infectious Diseases and Tropical Medicine Research Centre, Shahid Beheshti University of Medical Sciences, Tehran, IR Iran \\ ${ }^{2}$ University of Queensland Centre for Clinical Research, The University of Queensland, Brisbane, Australia \\ ${ }^{3}$ National research Institute of Tuberculosis and Lung Diseases, Shahid Beheshti University of Medical Sciences, Tehran, IR Iran \\ ${ }^{4}$ Department of Infectious Diseases and Tropical Medicine, Shahid Beheshti University of Medical Sciences, Tehran, IR Iran
}

\begin{tabular}{l}
\hline A R T I C L E I N F O \\
\hline Article type: \\
Case Report \\
\hline Article history: \\
Received: 30 Apr 2012 \\
Revised: 20 Jun 2012 \\
Accepted: 03 Jul 2012 \\
\hline
\end{tabular}

\begin{abstract}
A B S T R A C T
A few case reports of coinfections with Nocardia and Mycobacterium tuberculosis (TB) have previously been published in the literature. We report on a case of concomitant pulmonary nocardiosis and tuberculosis, in a recently diagnosed 55-year-old woman, with rheumatoid arthritis, who had been taking a short course of a low dose corticosteroid. The patient was treated with a combination of amikacin and imipenem, as well as a standard anti-TB regimen. Physiologic doses of corticosteroids might be a predisposing factor for opportunistic infections, such as nocardiosis or tuberculosis.
\end{abstract}

Keywords:

Nocardia

Mycobacterium tuberculosis

Corticosteroid

Pulmonary Disease

Published by Kowsar Corp, 2013. cc 3.0.

- Implication for health policy/practice/research/medical education:

Dual or multiple infections can be occurred in patients who are assumed not to be deeply immune compromised.

- Please cite this paper as:

Aminzadeh Z, Alavi Darazam I. Concomitant Pulmonary Nocardiosis and Tuberculosis in a Patient With Rheumatoid Arthritis: A New View point. Jundishapur J Microbiol. 2013;6(1):91-4. DOI:10.5812/jjm.5362

\section{Introduction}

Nocardia is a possible cause of pulmonary and systemic infection in immune-compromised patients; in addition, it has also been isolated in immune-competent patients. The annual incidence of nocardiosis in the United States is approximately 500-1 000 cases per year (1). Difficulties in isolating the organism, incubation experience and lack of systematic reports concerning No cardia infections in the health system, are probable reasons (2).
Predisposing conditions are; chronic obstructive pulmonary disease, transplantation, human immune deficiency virus (HIV) infection, alcoholism and taking steroids (3). Although a high dose of steroids has been considered to be a risk factor for opportunistic infections, a low maintenance dose of these agents in physiologic dosage usually does not take into account for these predisposing factors.

Herein, we report a rare and interesting case of concomitant pulmonary nocardiosis and Mycobacterium tu-

\footnotetext{
${ }^{*}$ Corresponding author: Ilad Alavi Darazam, National research Institute of Tuberculosis and Lung Diseases, Shahid Bahonar Ave, Darabad P.O:19575/154, Tehran, IR Iran.Tel:+98-9141491958, Fax:+21-20105050,E-mail: ilad13@yahoo.com

DOI:10.5812/jjm.5362

(C) 2013 Ahvaz Jundishapur University of Medical Sciences; Published by Kowsar Corp.

This is an Open Access article distributed under the terms of the Creative Commons Attribution License (http://creativecommons.org/licenses/by/3.0), which permits unrestricted use, distribution, and reproduction in any medium, provided the original work is properly cited.
} 
berculosis, in a middle-aged woman taking only a low dosage, short course of corticosteroids, in order to treat her newly diagnosed rheumatoid arthritis and undiagnosed bronchiectasis. We also reviewed all related cases recorded in well-known medical databases including Medline.

\section{Case History}

A woman in her fifties was admitted with a one month history of dyspnea, cough and copious sputum. She also complained of pleuritic chest pain in the left hemithorax without hempotysis, fever or chills. She had previously presented with three episodes of productive cough over the past five years, for which she took short courses of antibiotics. She had an unremarkable family and social history and had been healthy until one year ago, when she developed mild polyarthralgia and morning stiffness. Her symptoms had deteriorated over the previous three months during a trip to the north of Iran. Based on an elevated erythrocyte sedimentation rate (ESR), positive rheumatic factors (RF) and high levels of anti-cyclic citrullinated peptide (anti-CCP) antibody and other negative rheumatologic assays, she was diagnosed with rheumatoid arthritis. Prednisolone (7.5 mg/d), hydroxychloroquine once daily, celecoxib and weekly methotrexate (MTX) were administered. As a result of exertional dyspnea and a productive cough (two weeks later), she stopped taking MTX and celecoxib due to the progression in her respiratory symptoms. One week before admission, she was on ketotifen, $\mathrm{N}$-acetylcysteine (NAC), and planned prednisolone.

On arrival in emergency room, she appeared severely ill and she was in respiratory distress; respiratory rate 40 breaths $/ \mathrm{min}$, heart rate 120 beats $/ \mathrm{min}$, temperature $37.6^{\circ}$ $\mathrm{C}$, blood pressure $105 / 80 \mathrm{~mm} / \mathrm{Hg}$, and oxygen saturation 93\% (with 4 litres of mask oxygen). Facial flushing, oral thrush and musical lung sounds were the remarkable findings. Further investigation revealed leukocytosis (white blood cells: $17700 / \mathrm{mm}^{3}$ ), anemia (hemoglobin: $10.3 \mathrm{gr} / \mathrm{dL}$ ), normal renal and liver function tests, elevated ESR (110 mm/hr), elevated serum lactate dehydrogenase (LDH) (1190 U/L), and high random blood sugar (336 mg/ $\mathrm{dL})$. Mild proteinuria and glycosuria without pyuria, hematuria and casts were reported in the urinalysis. Chest roentgenogram showed multilobar infiltrates and also bronchiectasis, particularly in both of the upper lobes. She was commenced on azithromycin (500 mg loading dose, then $250 \mathrm{mg}$ daily), piperacillin-tazobactam (4.5 gr q6h), bronchodilator, and hydrocortisone (50 mg q3h). Her chest computed tomographic (CT) scan is shown in Figure 1.

After repeated negative acid fast staining of her sputum, she undertook a series of investigations including; echocardiography, bronchoscopy and bronchoalveolar lavage (BAL) sampling, and she was also evaluated for autoimmune diseases. The bronchoscopy revealed copious
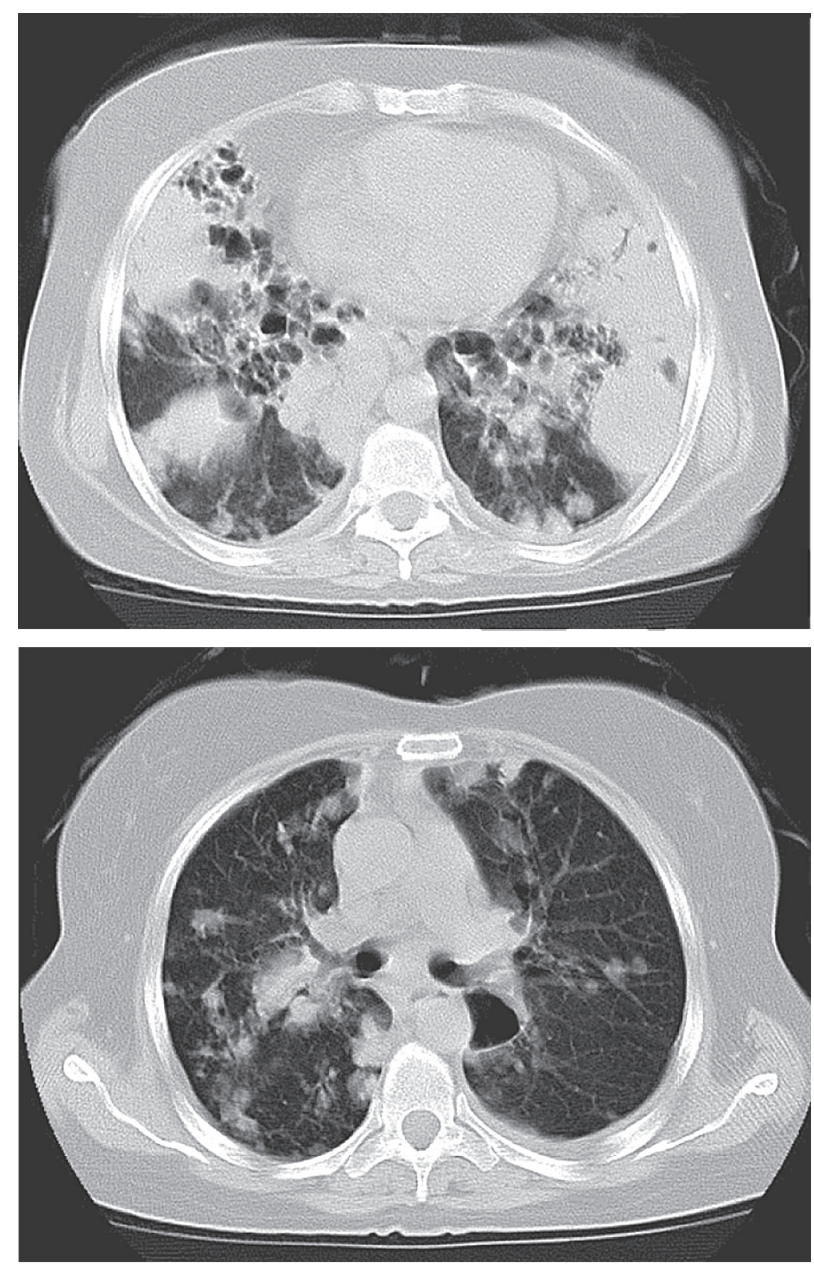

Figure 1. Computed Chest Tomography of Patient on Arrival Revealed Multiple Nodules and Discrete Alveolar Infiltration and Bronchiectasis.

secretions without endobronchial lesions. The echocardiography was normal; blood cultures showed no growth and early sputum cultures were also negative. Repeated high levels of anti-CCP and RF, elevated ESR, normal antinuclear antibodies (ANA), antineutrophil cytoplasmic antibodies (ANCA), anti-Ro, anti-La, anti-double stranded DNA (dsDNA), angiotensin convertase enzyme (ACE) and complements, along with a compatible history and clinical findings confirmed a diagnosis of rheumatoid arthritis (RA).

Eventually, a sputum culture revealed a heavy growth of Nocardia. Nocardia was also isolated from a BAL specimen culture after 48 hours. Therefore, piperacillin-tazobactam and azithromycin were discontinued and a combination of amikacin (10 mg/kg in two divided doses) with imipenem (500 mg q6h) was administered. On subsequent visits, she had made remarkable improvements. A normal brain CT scan excluded brain involvement. Three weeks after taking this antibiotic in combination with prednisolone (5 mg), hydroxychloroquine (once daily) and MTX (once weekly), the antibiotic regimen was changed to tri- 
methoprim/sulfamethoxazole (800 mg q12h) (Figure 2). Interestingly, the polymerase chain reaction (PCR) of the BAL specimen revealed $M$. tuberculosis, therefore, an anti-tuberculosis regimen (including isoniazid $300 \mathrm{mg} / \mathrm{d}$, rifampin $600 \mathrm{mg} / \mathrm{d}$, pyrazinamide $1000 \mathrm{mg} / \mathrm{d}$ and ethambutol 800 $\mathrm{mg} / \mathrm{d}$ ) was added to her previous medication; at follow-up, the BAL culture also confirmed the presence of $M$. tuberculosis. Three weeks after discharge, the patient complained of severe right shoulder pain and presented with zoster which was treated with three weeks of oral acyclovir (800 mg 5 times/d).

The evaluation of the probable underlying immune de-
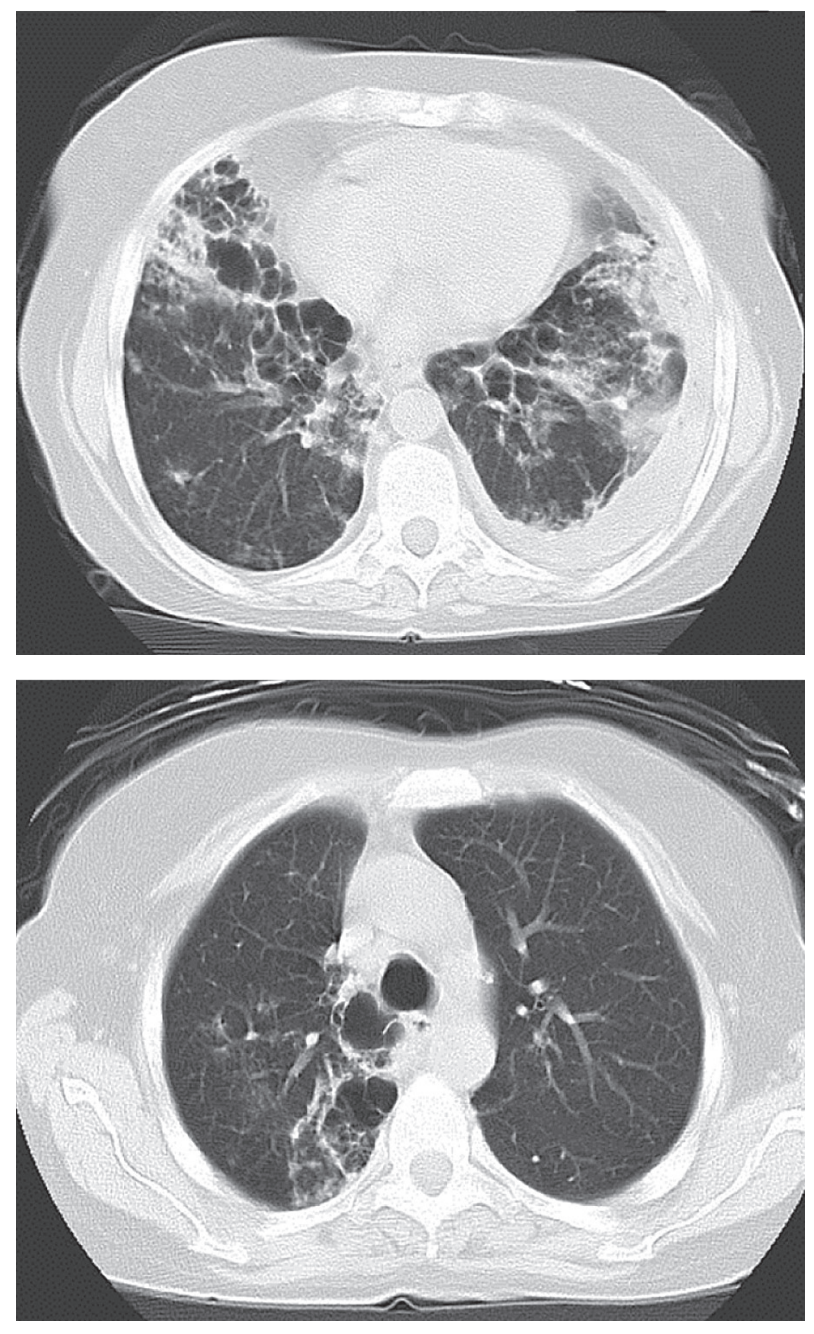

Figure 2. Computed Chest Tomography After Treatment of Nocardiosis Revealed Underlying Bronchiectasis With No Alveolar Infiltrations or Nodular Opacities.

ficiency, either the primary or the secondary, including white blood cell count and differentials, blood smear, peripheral lymphocytes flow cytometry, HIV Ab test, quantitative immunoglobulin and complement levels and nitroblue tetrazolium dye test (NBT) revealed no abnormalities. Our patient suffered from concomitant pul- monary nocardiosis and tuberculosis following a short course of low dose corticosteroids due to underlying rheumatoid arthritis and bronchiectasis.

\section{Discussion}

Phagocytic and cell mediated immunity play an important role against Nocardia infections, particularly in disseminated disease (4). Predisposing factors or underlying diseases have been found in $81 \%$ of nocardiosis patients (3).

Treatment with steroids is a strong predisposing factor for patients diagnosed with pulmonary nocardiosis (64.5\%) (3). However, a definite threshold of steroid dosage as a risk factor remains controversial $(5,6)$.

It seems that the overall rate of infectious complications was $12.7 \%$ in patients on systemic corticosteroids and $8.0 \%$ in the controls (relative risk: 1.6 (1.3-1.9). The rate was not increased in patients given a daily dose of less than $10 \mathrm{mg}$ or a cumulative dose of less than $700 \mathrm{mg}$ of prednisone (6). However, data from a prospective study revealed an increase in the frequency of all infections, bacterial and opportunistic infections in particular, which rose progressively with increasing steroid dose. In addition, the risk of infection rose from 1.5 fold at an average prednisone dose below $10 \mathrm{mg} /$ day, to over eight fold, in patients receiving doses above $40 \mathrm{mg} /$ day (5). Therefore, low dose corticosteroids may lead to immune suppression. In this case, the dose of corticosteroid (prednisolone) was $10 \mathrm{mg} / \mathrm{d}$ and symptoms were initiated in less than two weeks. Furthermore, it is known that rheumatoid arthritis significantly reduces cell-mediated immunity in comparison to controls (7).

Pulmonary tuberculosis associated with nocardiosis should be detected by various approaches. In the first place, pulmonary tuberculosis is a leading cause of lung parenchymal disruption. Certain notable cases of bronchiectatic lungs are due to previous pulmonary tuberculosis particularly in endemic areas. Furthermore, bronchiectasis is a known risk factor of pulmonary nocardiosis (4). Moreover, bronchiectasis may be a simultaneous predisposing condition associated with corticosteroids as shown in our patient. Pulmonary manifestations of nocardiosis are quite similar to pulmonary tuberculosis. Consequently, physicians in endemic regions may treat the patients with compatible symptoms of tuberculosis, despite negative acid fast staining of the sputum (8).

To determine the frequency of nocardiosis in HIV-positive individuals clinically suspected of having tuberculosis (TB), 140 sputum samples were analysed in African patients. Four (2.85\%) patients were positive for Nocardia by microscopy and five (3.6\%) had a positive culture for Nocardia asteroides. In areas where HIV-associated TB is common, some patients diagnosed as smear-negative pulmonary tuberculosis, might also suffer from nocardiosis (8).

Only a few case reports of concomitant infection of 
Nocardia and TB have been published in the literature (9). One of these included a 45-year-old woman with systemic lupus erythematosus (SLE), who presented with thoracic empyema that was unusually infected by $M$. tuberculosis. The second case was a 26-year-old HIV positive Thai man who presented with; progressive dyspnea for four months, right pleuritic chest pain and massive right pleural effusion, in which a thoracentesis revealed Nocardia as well as a positive strain for acid-fast bacilli of a nontuberculosis mycobacteria infection, concomitant with Nocardia asteroids. The third case was a 55-year-old man with diabetes mellitus who presented with infiltrative shadows in the right upper lung field (10-12). In the latter, repeated sputum smears showed no mycobacterium, so bronchoalveolar lavage (BAL) was performed bronchoscopically in the right bronchus at the right. The BAL fluid and the sputum obtained on the day after the BAL contained acid-fast, branching filamentous structures that were identified as Nocardia asteroides. Later, M. tuberculosis was detected in a six-week culture of the sputum and BAL fluid. Primary negative sputum for M. $t u$ berculosis and a late positive culture of the BAL fluid was identical to our case.

In 1 to $6 \%$ of patients with tuberculosis, Nocardia has also been isolated from the sputum (13). A total of 209 cases of pulmonary tuberculosis were screened for the presence of Nocardia in their sputa. In $2.9 \%$ of the cases Nocardia asteroides was isolated (14). The increasing importance of this microorganism has been discussed in the light of other reports from India (14). Of 1510 sputum samples cultured from 1016 patients in a tuberculosis and chest disease hospital in Amritsar, 67 sputa originating from 16 patients were found to be positive for the Nocardia asteroides species complex (a prevalence of $1.4 \%$ pulmonary). Of the various clinical categories of patients investigated, pulmonary tuberculosis with sputum negative for acidfast bacilli (AFB) yielded the highest prevalence of 3.2\%, followed by pneumonia, chronic obstructive pulmonary disease (COPD), bronchiectasis and pulmonary tuberculosis with sputum positive for AFB, at $1.3 \%, 1.2 \%, 1.1 \%$ and $0.5 \%$, respectively. The observations underscore the point that nocardiosis warrants greater attention in the differential diagnosis of bronchopulmonary diseases (15).

Interestingly, our case shows that underlying rheumatoid arthritis and bronchiectasis, in addition to a short course of low dose prednisolone, are possible predisposing factors of pulmonary nocardiosis and tuberculosis, this study reported such a rare concomitant infection.

\section{Conclusions}

To sum up, opportunistic infections such as nocardiosis can develop in patients taking corticosteroids regimens, even with a well-known 'physiologic dose'. Due to the possibility of concurrent infections, it is important to evaluate all possible causes of pulmonary involvement in patients with underlying disease.

\section{Acknowledgements}

We would like to thank the Microbiology Unit at the laboratory of the National Research Institute of Tuberculosis and Lung Diseases (NRITLD) and Dr. Payam Tabarsi for their assistance.

\section{Financial Disclosure}

None declared.

\section{Funding/Support}

None declared.

\section{Authors' Contribution}

None declared.

\section{References}

1. Beaman BL, Beaman L. Nocardia species: host-parasite relationships. Clin Microbiol Rev. 1994;7(2):213-64.

2. Beaman BL, Burnside J, Edwards B, Causey W. Nocardial infections in the United States, 1972-1974. J Infect Dis. 1976;134(3):286-9.

3. Alavi Darazam I, Shamaei M, Mobarhan M, Ghasemi S, Tabarsi P, Mansouri D. Nocardiosis: Risk factors, clinical characteristics and outcome. Iran Red Crescent Med J. 2012;In Press.

4. Forbes GM, Harvey FA, Philpott-Howard JN, O'Grady JG, Jensen $\mathrm{RD}$, Sahathevan M, et al. Nocardiosis in liver transplantation: variation in presentation, diagnosis and therapy. J Infect. 1990;20(1):11-9.

5. Ginzler E, Diamond H, Kaplan D, Weiner M, Schlesinger M, Seleznick $\mathrm{M}$. Computer analysis of factors influencing frequency of infection in systemic lupus erythematosus. Arthritis Rheum.1978;21(1):37-44.

6. Stuck AE, Minder CE, Frey FJ. Risk of infectious complications in patients taking glucocorticosteroids. Rev Infect Dis.1989;11(6):954-63.

7. Emery P, Panayi GS, Nouri AM. Interleukin-2 reverses deficient cell-mediated immune responses in rheumatoid arthritis. Clin Exp Immunol. 1984;57(1):123-9.

8. Rasheed MU, Belay G. Nocardiosis in HIV seropositive clinically suspected pulmonary tuberculosis patients. Trop Doct. 2008;38(1):34-5.

9. Chaudhury RC, Aher AR, Rastogi V, Prabhu TK. A case of mixed pulmonary infection by nocardia and Mycobacterium tuberculosis. Indian J Pathol Microbiol. 2009;52(2):294-5.

10. Huang HC, Yu WL, Shieh CC, Cheng KC, Cheng HH. Unusual mixed infection of thoracic empyema caused by Mycobacteria tuberculosis, nontuberculosis mycobacteria and Nocardia asteroides in a woman with systemic lupus erythematosus. J Infect. 2007;54(1):e25-8.

11. Inthraburan K, Wongsa A. Empyema thoracis due to nocardiosis and Mycobacterium tuberculosis mixed infections in an AIDS patient. Southeast Asian J Trop Med Public Health. 2009;40(4):776-80.

12. Shimokubo T, Ashitani J, Ihi T, Higa T, Kumamoto K. [A case of mixed infection by Nocardia asteroides and Mycobacterium tuberculosis]. Nihon Kokyuki Gakkai Zasshi. 2002;40(8):703-7.

13. Case records of the Massachusetts General Hospital. Weekly clinicopathological exercises. Case 11-1999. A 60-year-old woman with epidural and paraspinal masses. NEngl J Med.1999;340(15):1188-96.

14. Gupta ML, Grover PS, Bhardwaj BK. Nocardia associated with pulmonary tuberculosis. J Commun Dis. 1991;23(2):157-9.

15. Singh M, Sandhu RS, Randhawa HS, Kallan BM. Prevalence of pulmonary nocardiosis in a tuberculosis hospital in Amritsar, Punjab. Indian J Chest Dis Allied Sci. 2000;42(4):325-39. 